\title{
Exodontias múltiplas sob anestesia geral: relato de caso
}

Multiple exodonties under general anesthesia: case report

Múltiples exodonidades bajo anestesia general: reporte de caso Luiz Gustavo DA COSTA ${ }^{1}$

Alana Oswaldina Gavioli Meira DOS SANTOS ${ }^{2}$ José Carlos Garcia de MENDONÇA ${ }^{3}$

Julio Cesar Leite DA SILVA ${ }^{3}$

Gustavo Silva PELISSARO ${ }^{4}$

Albert Schiavetto DE SOUZA ${ }^{3}$

Ellen Cristina GAETTI-JARDIM ${ }^{3}$

${ }^{1}$ Cirurgião-Dentista. Faculdade de Odontologia da Universidade Federal de Mato Grosso do Sul (Faodo-UFMS)

${ }^{2}$ Residente em Cirurgia e Traumatologia Bucomaxilofacial da Faculdade de Odontologia da Universidade Federal de Mato Grosso do Sul (Faodo-UFMS).

${ }^{3}$ Docente. Universidade Federal de Mato Grosso do Sul (Faodo-UFMS).

${ }^{4}$ Doutorando. Universidade Federal de Mato Grosso do Sul (Famed-UFMS)

\section{Resumo}

O tratamento odontológico de pacientes oncológicos é uma realidade. A osteorradionecrose durante muito tempo foi considerada como um tipo de osteomielite do osso irradiado, sendo uma das mais importantes complicações da radioterapia de cabeça e pescoço complicação ainda se faz presente apesar dos avanços no tratamento do paciente com câncer. Deste modo, é objetivo deste, apresentar um caso de paciente com diagnóstico de câncer em região de cabeça e pescoço com necessidade de exodontias múltiplas prévias ao tratamento radioterápico e de quimioterapia. Paciente de 50 anos de idade, sexo masculino, procurou atendimento na Faculdade de Odontologia da Universidade Federal de Mato Grosso do Sul, encaminhado de dentista particular para exodontia múltipla apresentando ao exame intrabucal dentes com mobilidade e inúmeras cáries e raízes residuais o mesmo confirmado pelo exame ortopantomográfico. Sendo assim, o plano de tratamento foi de exodontia de 25 elementos associado a regularização de rebordo, sob anestesia geral. Após internação hospitalar, exames de rotina e o procedimento sob anestesia geral o paciente recebeu alta sem queixas, com medicações analgésicas e retorno programado. A terapêutica se mostrou satisfatória e a resposta do paciente ao tratamento também o que configura sucesso.

Descritores: Cirurgia Bucal; Osteorradionecrose; Anestesia Geral.

\section{Abstract}

The dental treatment of cancer patients is a reality. Osteorradionecrosis has long been considered as a type of osteomyelitis of the irradiated bone, being one of the most important complications of head and neck radiotherapy. This complication is still present despite advances in the treatment of cancer patients. Thus, it is the objective of this study to present a case of a patient with a diagnosis of cancer in the head and neck region with the need for multiple exodontia prior to radiotherapy and chemotherapy. A 50-year-old male patient sought care at the Faculty of Dentistry of the Federal University of Mato Grosso do Sul, referred by a private dentist for multiple exodontia, presenting with intrabucal examination teeth with mobility and innumerable cavities and residual roots, which was confirmed by the ortopantomographic exam. Therefore, the treatment plan was 25 -fold extraction associated with regularization of the ridge, under general anesthesia. After hospitalization, routine exams and the procedure under general anesthesia the patient was discharged without complaints, with analgesic medications and scheduled return. Therapy was satisfactory and the patient's response to treatment was also successful.

Descriptors: Oral Surgery; Osteoradionecrosis; General Anesthesia.

\section{Resumen}

El tratamiento dental de pacientes con cáncer es una realidad. La osteorradionecrosis se ha considerado durante mucho tiempo como un tipo de osteomielitis ósea irradiada, una de las complicaciones más importantes de la radioterapia de cabeza y cuello, aún presente a pesar de los avances en el tratamiento de pacientes con cáncer. Por lo tanto, el objetivo de este trabajo es presentar un caso de un paciente diagnosticado con cáncer de cabeza y cuello que requirió extracciones múltiples antes del tratamiento de radioterapia y quimioterapia. Un paciente masculino de 50 años buscó tratamiento en la Facultad de Odontología de la Universidad Federal de Mato Grosso do Sul, derivado de un dentista privado a extracción múltiple, presentando los dientes con movilidad y numerosas cavidades y raíces residuales confirmadas por el examen. ortopantomografico. Por lo tanto, el plan de tratamiento fue la extracción de 25 elementos asociada con la regularización del labio bajo anestesia general. Después de la hospitalización, los exámenes de rutina y el procedimiento bajo anestesia general, el paciente fue dado de alta sin quejas, con medicamentos analgésicos y regreso programado. La terapia fue satisfactoria y la respuesta del paciente al tratamiento también fue un éxito.

Descriptores: Cirugía Oral; Osteorradionecrosis; Anestesia General.

\section{INTRODUÇÃO}

Segundo o Instituto Nacional de Câncer (INCA) estimam-se 14.700 novos casos de câncer de cavidade oral dentre homens e mulheres para os anos do biênio 2018-2019. Isso corresponde a um risco de 10,86 novos casos a cada 100 mil homens e de 3,28 para cada 100 mil mulheres, o colocando na quinta posição dentre homens e decima segunda dentre as mulheres ${ }^{1}$.

O tratamento do câncer é realizado através de três maneiras: a cirurgia, a quimioterapia e a radioterapia, podendo estas serem utilizadas individualmente ou associadas, dependendo das características da neoplasia a ser tratada ${ }^{2-6}$. Dos três, o tratamento cirúrgico é o único que pode atuar diretamente no tecido doente. Já a quimioterapia e a radioterapia atuam inibindo ou destruindo as células, não fazendo diferenciação entre as células neoplásicas e as células saudáveis do corpo. Desta forma há uma maior chance de haver efeitos colaterais secundários ${ }^{5,7}$.

Tanto a quimioterapia como a radioterapia podem gerar severas alterações na mucosa bucal ${ }^{5,8}$. Reações adversas como mucosite, xerostomia, candidíase, disgeusia, osteoradionecrose dentre outros, que afetam a qualidade de vida dos pacientes, podendo acarretar problemas em seu tratamento. Isso depende da frequência e da dose em que é realizada a radioterapia, do local irradiado, da idade e condições clínicas do paciente e dos tratamentos associados, sendo que as manifestações podem ser agudas (pouco 
tempo após o tratamento) ou crônicas (podendo se manifestar de meses a anos após o tratamento $)^{9}$.

Isto posto, a preparação de um plano de tratamento detalhado é de suma importância para pacientes oncológicos, de modo a minimizar os riscos de desenvolvimento de manifestações de sequelas, sobretudo da radiação. Dessa forma, o atendimento odontológico pregresso se torna fundamental, no intuito de gerar maior qualidade de vida ao paciente, durante e, sobretudo após o incremento da terapêutica oncológica $^{10,11}$.

\section{CASO CLÍNICO}

Paciente do sexo masculino, 50 anos de idade, compareceu a consulta na Faculdade de Odontologia (FAODO) da Universidade Federal de Mato Grosso do Sul com encaminhamento para remoção dos elementos dentários previamente a tratamento de radioterapia e quimioterapia.

Ao exame clínico extrabucal foi observado aumento de volume em região cervical esquerda. Ao exame intra-bucal, dentes 16, 11, 21, 22, 23, 24 e 44 com grande destruição coronária, dentes 18, 17, 27, $28,12,31,32,32,34,35,41,42,43,45$ e 48 com grande comprometimento periodontal. Ao exame de imagem, ortopantomografia, foi observada a necessidade também de extração do elemento 38 que estava mesializado e dos elementos 13,14 e 15 (Figura 1).
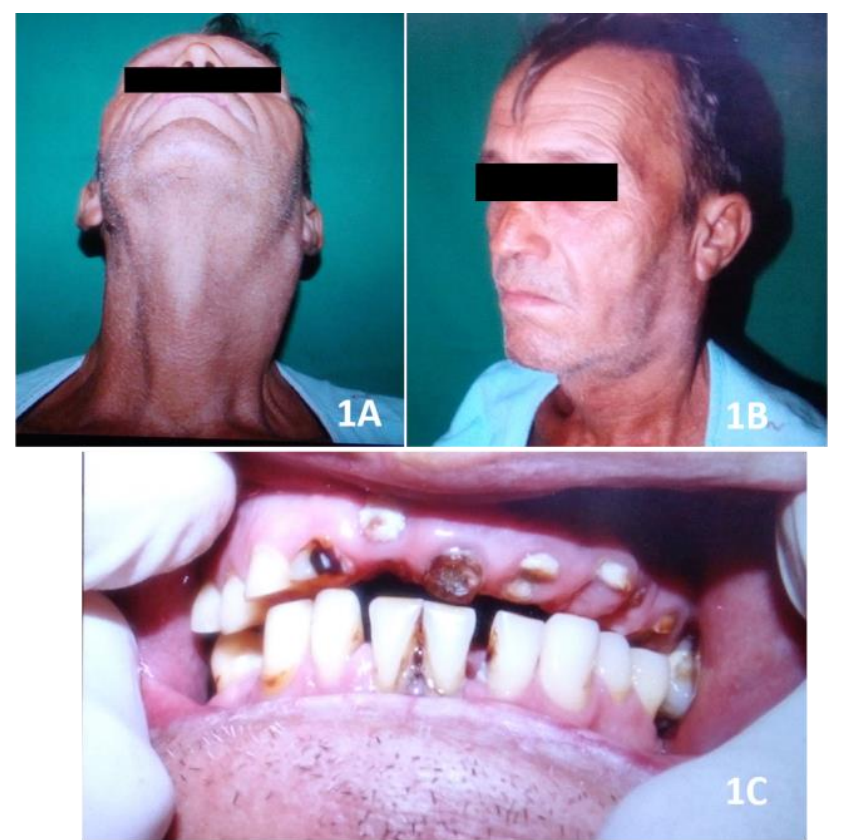

Figura 1: Aspecto clínico extra e intrabucal. Abaulamento em região cervical a esquerda associado a necessidade de exodontias múltiplas.

Ao atendimento inicial foi realizada a abertura de ficha clínica e coleta dos dados como idade, sexo, condição sistêmica com diagnóstico oncológico assim como a presença do encaminhamento de dentista particular e do médico oncologista solicitando a intervenção odontológica. Deste modo, após análise dos dados acima bem como do diagnóstico odontológico, constatada a necessidade de exodontia total, 25 elementos dentários, a fim de otimizar o tratamento com o oncologista, optou-se pelo tratamento em nível hospitalar e sob anestesia geral para exodontia em sessão única. Sendo assim, em virtude dos procedimentos numerosos e da urgência do paciente já com laudo e encaminhamento de oncologista para adequação do meio bucal previamente ao tratamento radio e quimioterápico para o carcinoma epidermoide cervical o paciente foi submetido a internação hospitalar para realização de procedimento sob anestesia geral. $\mathrm{O}$ paciente foi submetido a avaliação sistêmica pela equipe médica de cirurgia de cabeça e pescoço e de anestesiologia bem como exames de laboratório com material sangue, sendo liberado para o procedimento odontológico. Foi realizado préoperatório de clindamicina 600mg 01 hora antes do procedimento, endovenoso, para profilaxia antibiótica bem como dexametasona 10mg também endovenoso. No centro cirúrgico, o paciente realizou antissepsia intra-oral com bochecho de digluconato de clorexidina a $0,12 \%$ durante 1 minuto. Após indução da anestesia geral e intubação nasotraqueal foi realizada antissepsia extraoral com solução de Clorexidina em região Peri-oral e labial, aposição de campos estéreis, colocação de tampão em orofaringe, seguida de infiltração anestésica em fundo de fórnix maxilar e mandibular a fim de prover um campo enxágue. Fez-se posteriormente a exodontia total dos dentes mencionados acima com fórceps e extratores a pressão manual. Irrigação abundante com soro fisiológico $0,9 \%$ e sutura dos alvéolos com pontos interrompidos de nylon 5-0. Após, paciente foi submetido a extubação e seguiu aos cuidados do profissional da anestesia (Figura 2).

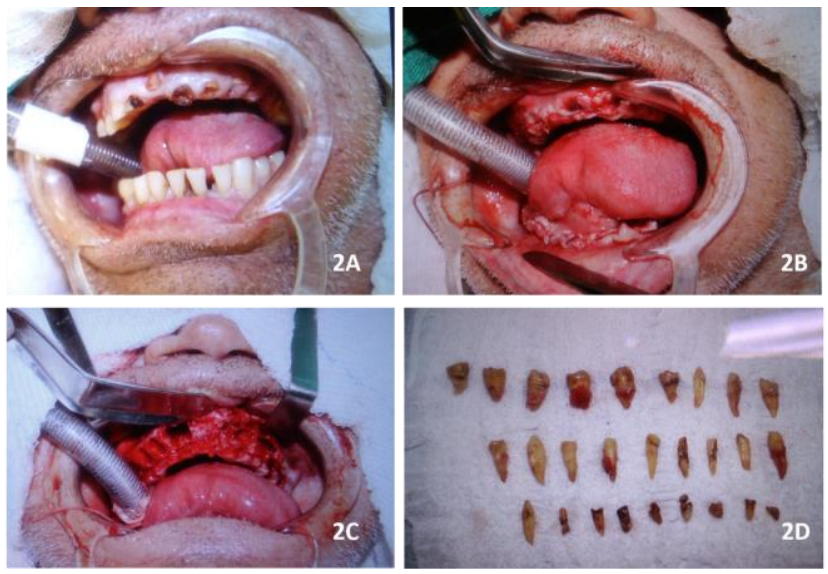

Figura 2: Aspecto clínico trans-operatório sob anestesia geral. Exodontia total e regularização de rebordo, com presença de intubação orotraqueal em posição.

Uma hora após foi encaminhado ao setor de enfermaria para realização de medicação analgésica (dipirona 500mg endovenoso 6/6 horas) e realização de exame radiográfico pós-operatório. Paciente se manteve sem queixas e com bom aspecto das abordagens no pós-operatório inicial bem como 24 horas e 48 horas após, recebendo assim, alta 
hospitalar com encaminhamento para avaliação pósoperatória tardia, 7 dias após o procedimento. Em pós-operatório de 07 dias, paciente apresentou-se sem queixas álgicas e ou funcionais, ausência de sinais flogísticos e boa cicatrização em maxila e em mandíbula.

\section{DISCUSSÃO}

Segundo a Organização mundial da saúde (OMS) o câncer é um termo genérico para um grupo de doenças que podem afetar qualquer parte de nosso corpo. Sua principal característica é a rápida proliferação de celular anormais, que excedem os seus limites habituais, podendo invadir partes distintas do corpo e se propagar pelo mesmo ${ }^{12}$.

As principais formas de tratamento do câncer são a cirurgia, a quimioterapia e a radioterapia de maneira individual ou associadas ${ }^{2,6,13}$. Dentre os variados tipos de câncer, o câncer de cavidade oral tem prevalência de $5,2 \%$ dos novos casos para homens e $1,7 \%$ para mulheres ${ }^{14}$. O câncer labial é a lesão maligna mais frequente da cavidade oral, sendo o carcinoma de células escamosas o mais prevalente ${ }^{15}$. As formas terapêuticas mais comuns no tratamento desse tipo de neoplasia maligna é a radioterapia de cabeça e pescoço e a cirurgia ${ }^{4}$. Sendo assim, são comumente encontradas uma série de complicações, das terapêuticas oncológicas, na cavidade bucal, que poderiam ser minimizadas se $o$ tratamento odontológico prévio fosse realizado o que torna o essencial para produzir o mínimo de qualidade de vida ao paciente.

Dentre tais complicações, as de maior interesse ao cirurgião dentista estão a mucosite, a infecção bucal oportunista, a função glandular alterada, a hiposalivação, a xerostomia, a doença periodontal, a carie de radiação, o trismo, a disfagia, a disgeusia, a candidose, a radiodermite, a hipogeusia e a osteorradionecrose $e^{4,16,17}$. Sendo que esta última poderia ser evitada se a exodontia prévia ao tratamento radioterápico pois após o tratamento de radiação o tecido se torna hipóxico, hipovascular e hipocelular prejudicando neoformação óssea, favorecendo a osteorradionecrose ${ }^{4,18}$. Ela se apresenta clinicamente como ulceração da mucosa, exposição óssea e presença de sintomatologia dolorosa acometendo em maior incidência a mandíbula ${ }^{4,18}$.

É preciso realizar uma adequação do meio bucal previamente ao tratamento oncológico (no mínimo três semanas antes de seu inicio), com o objetivo de eliminar ou estabilizar as condições bucais, para minimizar a infecção local e sistêmica, durante e após o tratamento do câncer ${ }^{19}$. Este tratamento inclui: exodontias (técnica cirúrgica menos atraumática possível, com remoção de espículas ósseas); tratamento endodôntico; remoção das lesões de cárie e selamento com ionômero de vidro, assim como a troca das restaurações insatisfatórias; correções de próteses mal adaptadas; tratamento periodontal para permitir suficiente cicatrização; orientação ao paciente quanto ao controle de placa, uso de escovas macias, pastas fluoretadas, uso de fio dental e escovas interproximais ${ }^{20}$, tais considerações levam a crer na necessidade eminente da comunicação do cirurgiãodentista com o médico oncologista a fim de juntos incrementarem qualidade de vida ao paciente em tratamento.

É necessário que a equipe odontológica seja incluída no tratamento desde seu inicio, a fim de permitir os cuidados necessários para evitar lesões em boca. Na avaliação odontológica deve ser considerado o histórico médico do paciente, somado a um exame odontológico minucioso, a fim de evitar problemas vindouros e conter infecções já estaladas na cavidade bucal ${ }^{19}$. Além disso, deve-se reforçar as instruções de higiene oral ao paciente, a fim de possibilitar um autocuidado satisfatório no trans e pós tratamento oncológico.

No caso relatado neste presente estudo foi constatada a necessidade de urgência no tratamento oncológico. Isto devido ao estagio de evolução do carcinoma espinocelular, localizado na porção posterior da língua, acometendo também a orofaringe do paciente. Por tanto, havia a necessidade de rapidez no tratamento odontológico do paciente, adequando a cavidade bucal para receber incidência de radiação terapêutica.

A partir da constatação desta necessidade, foi decidido levar a assistência odontológica ao ambiente hospitalar, sob anestesia geral, sendo esta uma das indicações de sedação total do paciente, uma vez que através da anestesia geral é possível realizar a reabilitação oral total numa única sessão, realizando desde profilaxias a cirurgias ${ }^{21}$. Dentre as indicações de uso de anestesia geral estão a necessidades de tratamentos múltiplos em portadores de doenças sistêmicas e pacientes especiais que necessitam de atendimento odontológico imediato ${ }^{22,23}$. Dessa forma, a adequação do meio foi realizada por meio de múltiplas extrações dentárias sob efeito de anestesia geral em ambiente controlado.

CONCLUSÃO

Baseado no caso exposto, a terapêutica mostrou sucesso e condizente com o quadro do paciente. A necessidade do atendimento e adequação do meio bucal de pacientes oncológico é fundamental para a busca de maior qualidade de vida a esses pacientes, minimizando ou mesmo evitando possíveis efeitos deletérios do tratamento para o câncer.

\section{REFERÊNCIAS}

1. INCA - Instituto Nacional de Câncer - Estimativa 2018 - Síntese de Resultados e Comentários [Internet]. 2018 [citado 27 de maio de 2019]. 
Disponível

em:

http://www1.inca.gov.br/estimativa/2018/sintesede-resultados-comentarios.asp

2. Bertrand J, Luc B, Philippe M, Philippe P. Anterior mandibular osteotomy for tumor extirpation: a critical evaluation. Head Neck. 2000;22(4):323-27.

3. Eisen MD, Weinstein GS, Chalian A, Machtay M, Kent K, Coia LR, et al. Morbidity after midline mandibulotomy and radiation therapy. Am J Otolaryngol. 2000;21(5):312-17.

4. Freitas DA, Caballero AD, Pereira MM, Oliveira SKM, Silva GPE, Hernández CIV. Sequelas bucais da radioterapia de cabeça e pescoço. Rev CEFAC. 2011;13(6):1103-8.

5. Hespanhol FL, Tinoco EMB, Teixeira HG de C, Falabella MEV, Assis NM de SP. Buccal manifestations in patients submitted to chemotherapy. Ciênc saúde coletiva. 2010; 15(Suppl 1):1085-94.

6. Shah JP, Gil Z. Current concepts in management of oral cancer-surgery. Oral Oncol. 2009; 45(4-5):394-401.

7. Santos FC. Tratamento odontológico em pacientes com câncer: revisão sistemática [monografia]. Natal: Universidade Federal do Rio Grande do Norte - UFRN; 2014.

8. Jesus LG de, Cicchelli M, Martins GB, Pereira MCC, Lima HS, Medrado ARAP. Repercussões orais de drogas antineoplásicas: uma revisão de literatura. RFO UPF. 2016;21(1):130-35.

9. Specht L. Oral complications in the head and neck radiation patient. Introduction and scope of the problem. Support Care Cancer. 2002;10(1):36-9.

10.Epstein JB, Thariat J, Bensadoun RJ, Barasch A, Murphy BA, Kolnick L et al. Oral complications of cancer and cancer therapy: from cancer treatment to survivorship. CA Cancer J Clin. 2012;62(6):400-22.

11.Samonis G, Mantadakis E, Maraki S. Orofacial viral infections in the immunocompromised host. Oncol Rep. 2000;7(6):1389-94.

12.OMS. Cáncer. Disponível em: https://www.who.int/es/news-room/factsheets/detail/cancer

13. Klein J, Livergant J, Ringash J. Health related quality of life in head and neck cancer treated with radiation therapy with or without chemotherapy: a systematic review. Oral Oncol. 2014;50(4):254-62.

14.Inca. Estimativa 2018: incidência de câncer no Brasil / Instituto Nacional de Câncer José Alencar Gomes da Silva. Coordenação de Prevenção e Vigilância. - Rio de Janeiro: INCA, 2017.

15. Kornalewsk FZ, Rodrigues SA de L, Lara P de LP, Accioly MCM, Haack AG, Kornalewsk FZ, et al. Abordagem de via aérea difícil em paciente com extenso tumor em lábio inferior - relato de caso. Anestesia Analgesia Reanimación. 2017;30(SPE):61-61.

16. Salazar M, Victorino FR, Paranhos LR, Ricci ID, Gaetti WP, Caçador NP. Efeitos e tratamento da radioterapia de cabeça e pescoço de interesse ao cirurgião dentista - revisão da literatura. Odonto. 2009;16(31):62-8.

17.Bueno CA, Magalhães CS, Moreira AN. Associações entre fatores de risco e complicações bucais em pacientes com câncer de cabeça e pescoço tratados com radioterapia associada ou não à quimioterapia. Pesq Bras Odontoped Clín Int. 2012;12(2):187-93.

18.Santos R dos, Dall'Magro AK, Giacobbo J, Lauxen JR, Dall'Magro E. Osteorradionecrose em pacientes submetidos à radioterapia de cabeça $\mathrm{e}$ pescoço: relato de caso. RFO UPF. 2015;20(2):232-37.

19.Vieira DL, Leite AF, Melo NS, Figueiredo PTS. Tratamento odontológico em pacientes oncológicos. Oral Sciences. 2012;4(2):37-42.

20.Santos CC, Noro-Filho GA, Caputo BV, Souza R, de Souza RC, de Andrade CMR, Giovani EM. Condutas práticas e efetivas recomendadas ao cirurgião dentista no tratamento pré, trans e pós do câncer bucal. J Health Sci Inst. 2013;31(4):368-72.

21.Lee P-Y, Chou M-Y, Chen Y-L, Chen L-P, Wang C-J, Huang W-H. Comprehensive dental treatment under general anesthesia in healthy and disabled children. Chang Gung Med J. 2009;32(6):636-42.

22. American Academy of Pediatric Dentistry. Clinical guideline on the elective use of minimal, moderate, and deep sedation and general anesthesia for pediatric dental patients. Pediatr Dent. 2004;26(7 Suppl):95-103.

23. Santos JS, Valle DA, Palmier AC, Amaral JHL do, Abreu MHNG de, Santos JS, et al. Utilização dos serviços de atendimento odontológico hospitalar sob sedação e/ou anestesia geral por pessoas com necessidades especiais no SUS-MG, Brasil. Ciênc saúde coletiva. 2015;20(2):515-24. 
http://dx.doi.org/10.21270/archi.v8i12.4800

\section{CONFLITO DE INTERESSES}

Os autores declaram não haver conflitos de interesse.

\section{AUTOR PARA CORRESPONDENCIA}

Alana Oswaldina Gavioli Meira dos Santos

nagavioli@gmail.com

Submetido em 12/09/2019

Aceito em 31/12/2019 